\title{
Liderazgo sistémico en las competencias gerenciales docentes universitarias
}

\section{Systemic leadership in competencesmanagers university teachers}

\author{
Julio Aldana \\ julioaldanazavala@gmail.com \\ Universidad Nacional Experimental Francisco de Miranda \\ Venezuela \\ https://orcid.org/0000-0002-7934-9103 \\ Mariannys Salón \\ mariannys.mmsp@gmail.com \\ Universidad Nacional Experimental Francisco de Miranda \\ Venezuela \\ Nelson Guzmán \\ mariannys.mmsp@gmail.com \\ Universidad Nacional Experimental Francisco de Miranda \\ Venezuela
}

\section{RESUMEN}

El liderazgo sistémico como eje integrador de las competencias gerenciales en la Universidad Nacional Experimental Francisco de Miranda (UNEFM), representa una alternativa epistémica emergente para coadyuvar teóricamente en la comprensión de un fenómeno que tiende a ser utilizado con mayor frecuencia en las organizaciones, por cuanto permite trabajar sistemática y sinérgicamente entre los miembros de la misma, hasta convertirse en un equipo de trabajo que transita hacia lo "inteligente", es decir, aprenden constantemente desde sus propias fortalezas y potencialidades para generar praxis gerenciales con base en un liderazgo integrador en procura de obtener la mayor efectividad posible en el cumplimiento de las metas propuestas. De allí que el propósito principal sea "generar reflexiones sobre los hallazgos del liderazgo sistémico del docente como eje integrador de las competencias gerenciales en la Universidad 
Nacional Experimental Francisco de Miranda". Ante lo cual se trabaja con un diseño cualitativo de investigación, sustentando en el paradigma interpretativo y método hermenéutico. Desde las vivencias, experiencias, de los informantes claves, se develarán los significados otorgados al liderazgo sistémico como un ente motivador y generador de conocimientos para aprender haciendo y consolidar la organización hacia una visión integradora de la realidad en procura de una gerencia que fomente las potencialidades de las personas hacia el logro de las metas comunes de la organización, bajo premisas de eficacia, calidad. Es de destacar que esta es una investigación en construcción, ante lo cual, no existen aportes finales, sino, la visión teleológica de los investigadores.

Palabras Claves: Pensamiento sistémico. Líder sistémico. Competencias gerenciales

\begin{abstract}
Systemic leadership as an integrating axis of managerial competencies at the Francisco de Miranda National Experimental University (UNEFM), represents an emerging epistemic alternative to theoretically contribute to the understanding of a phenomenon that tends to be used more frequently in organizations, as allows to work systematically and synergistically among its members, until it becomes a work team that moves towards the "intelligent", that is, they constantly learn from their own strengths and potentials to generate managerial praxis based on an integrative leadership in to obtain the greatest possible effectiveness in meeting the proposed goals. Hence, the main purpose is "to generate reflections on the findings of the systemic leadership of the teacher as an integrating axis of management skills at the National Experimental University Francisco de Miranda." In view of this, we work with a qualitative research design, based on the interpretive paradigm and hermeneutical method. From the experiences, experiences, of the key informants, the meanings given to the systemic leadership will be revealed as a motivating entity and generator of knowledge to learn by making and consolidating the organization towards an integrating vision of the reality in search of a management that promotes the potentialities of people towards the achievement of the common goals of the organization, under premises of effectiveness, quality. It is noteworthy that this is a research under construction, before which, there are no final contributions, but the teleological vision of researchers.
\end{abstract}

Key Words: Systemic thinking. Systemic leader Managerial competencies 


\section{CIENCIAMATRIA \\ Revista Interdisciplinaria de Humanidades, Educación, Ciencia y Tecnología \\ Año V. Vol. V. N8. Enero - Junio 2019 \\ Hecho el depósito de ley: pp201602FA4721 \\ ISSN-L: 2542-3029; ISSN: 2610-802X \\ Universidad Nacional Experimental Francisco de Miranda (UNEFM). Santa Ana de Coro. Venezuela \\ Julio Aldana; Mariannys Salón; Nelson Guzmán}

\section{INTRODUCCIÓN}

Las organizaciones educativas se encuentran ante el desafío de transcender a nuevos modelos de liderazgo y de gerencia, tanto en los ambientes de aprendizaje como a nivel directivo, por cuanto el docente es un líder, gerente, en todos los contextos donde se desenvuelva. La gerencia educativa tiene el reto de ser cada día más eficiente, para trabajar organizadamente en la consecución de una educación de calidad que brinde repuesta a las múltiples necesidades y aspiraciones de la sociedad globalizada, para esto se hace necesario trabajar con una visión multidisciplinar en la gerencia educativa, esto con el fin de potenciar las habilidades y competencias de las personas que forman parte de la institución educativa, fomentado así, lo mejor de sí al cumplimiento de metas propuestas por la institución.

Es necesario que directivos, docentes, estudiantes, personal administrativo, obrero, padres, representantes, comunidad, se integren para generar la sinergia necesaria para lograr la educación de calidad cónsona con los retos socio productivo de la sociedad venezolana. Para esto es necesario que los docentes directivos trabajen con perspectiva integral sobre el direccionamiento al cual deben orientar la institución, desde un punto de vista gerencial, para esto es necesario transcender los modelos autocráticos, verticales, de liderazgo y gerencia con el cual, tradicionalmente se ha trabajado, debido que la sociedad actual, demanda mayor integración desde la complejidad en la cual se desarrolla la misma.

De allí que en los últimos tiempos, se ha venido proyectando el liderazgo sistémico como eje integrador de nuevas praxis para la consolidación de un liderazgo sinérgico transformador, siendo necesario conocer desde los actores que hacen vida en las instituciones universitarias, como vivencian, comprenden, lo relacionado al liderazgo docente y sí de él se deriva o se trabaja el sistémico.

Conocer esta realidad dinámica implicará replantearse el modo de ser líder - gerente en el contexto educativo universitario, lo cual podrá teorizarse como un elemento emergente en consonancia para coadyuvar a la retroalimentación teórica - bibliográfica 


\section{CIENCIAMATRIA \\ Revista Interdisciplinaria de Humanidades, Educación, Ciencia y Tecnología \\ Año V. Vol. V. N8. Enero - Junio 2019 \\ Hecho el depósito de ley: pp201602FA4721 \\ ISSN-L: 2542-3029; ISSN: 2610-802X \\ Universidad Nacional Experimental Francisco de Miranda (UNEFM). Santa Ana de Coro. Venezuela \\ Julio Aldana; Mariannys Salón; Nelson Guzmán}

venezolana, situación que aportaría bases al conocimiento colectivo del mundo educativo, es por esa razón que en el presente avance inicial de una investigación que se encuentra en construcción, se reflexiona sobre el "liderazgo sistémico del docente como eje integrador de las competencias gerenciales en el contexto universitario".

\section{Propósito principal de la investigación}

Generar reflexiones sobre los hallazgos del liderazgo sistémico del docente como eje integrador de las competencias gerenciales en la Universidad Nacional Experimental Francisco de Miranda.

\section{Propósitos secundarios de la investigación}

- Develar el sentido del liderazgo sistémico del docente como eje integrador de las competencias gerenciales.

- Construir las categorías emergentes sobre el liderazgo sistémico del docente y las competencias gerenciales.

- Interpretar los significados que configuran el liderazgo sistémico del docente como eje integrador de las competencias gerenciales.

\section{REFERENCIALES TEÓRICOS INICIALES}

\section{Pensamiento sistémico}

El pensamiento sistémico, aborda la interconexión del ser humano con el mundo, para la resolución de conflictos con el fin de mejorar las relaciones interpersonales y acciones que se desarrollan en las organizaciones. En este sentido, Senge (2006: 8), señala que

El pensamiento sistemático es un conjunto de técnicas y herramientas en los cuales abarca dos conceptos principales: realimentación y servomecanismos que han servido de ayuda para comprender a los sistemas empresariales. Es una disciplina para ver las estructuras que subyacen a las situaciones 


\section{CIENCIAMATRIA \\ Revista Interdisciplinaria de Humanidades, Educación, Ciencia y Tecnología \\ Año V. Vol. V. N8. Enero - Junio 2019 \\ Hecho el depósito de ley: pp201602FA4721 \\ ISSN-L: 2542-3029; ISSN: 2610-802X \\ Universidad Nacional Experimental Francisco de Miranda (UNEFM). Santa Ana de Coro. Venezuela \\ Julio Aldana; Mariannys Salón; Nelson Guzmán}

complejas y para discernir cambios de alto y bajo apalancamiento, resumiéndolo a la restructuración de nuestro pensamiento.

Partiendo de la cita planteada, el pensador sistémico tiene la capacidad de reflexionar en su accionar personal - profesional sobre el rumbo que debe afrontar para ser mejor persona y trabajador, entiende que es parte de un sistema y que debe aportar lo mejor de sí para que la organización pueda enfocarse al éxito continuo. Por consiguiente, el pensamiento sistemático permite tener la capacidad de generar información, con el fin de estimular la interdependencia y para promover transformaciones sinérgicas en la institución. Por consiguiente, algunos elementos del pensamiento sistemático son: que modela sistemas complejos, observa el todo, reduce problemas y situaciones complejas, abre nuestra mente y es un modelo que tiene un leguaje circular.

Es así que la metodología desarrollada en el liderazgo sistémico, es abordada desde las siguientes vertientes: 1 . Visión global en el cual se puede observar todo de manera general. 2. Balance del corto y largo plazo en el cual se lleva a través del camino del éxito y va a depender de las implicaciones que se presente. 3 . Reconocimiento de los sistemas dinámicos complejos e interdependientes en el cual a través de herramientas se construyen modelos para las situaciones bajo observación. 4. Reconocimiento de los elementos medibles y no medibles el cual el pensamiento sistemático impulsa el correcto uso de indicadores cualitativos y cuantitativos a través de un análisis de la situación.

De ese modo, el pensamiento sistémico es contextualizado en la realidad organizacional con el fin de generar planes de acción que permitan enfocarse al desarrollo eficaz y productivo de la organización, en donde se puedan abordar estrategias que permitan proyectar al éxito continuo a las personas que hacen vida en la institución desde una visión de trabajo en equipo, generando la sinergia necesaria para lograr las metas comunes que impulsen el desarrollo de la gestión organizacional. 


\section{CIENCIAMATRIA \\ Revista Interdisciplinaria de Humanidades, Educación, Ciencia y Tecnología \\ Año V. Vol. V. N8. Enero - Junio 2019 \\ Hecho el depósito de ley: pp201602FA4721 \\ ISSN-L: 2542-3029; ISSN: 2610-802X \\ Universidad Nacional Experimental Francisco de Miranda (UNEFM). Santa Ana de Coro. Venezuela \\ Julio Aldana; Mariannys Salón; Nelson Guzmán}

\section{Liderazgo sistémico}

El liderazgo sistémico es un nuevo estilo de gerenciar en las organizaciones, implica trabajar el todo sin descuidar sus partes, en este sentido, Medina, Nava y Bong (2010:

5) señalan que

La esencia del liderazgo sistémico proviene de la sinergia organizacional. Este enfoque se basa en el desarrollo en conjunto de manera simultánea, tanto para los factores internos como los externos, es necesario desarrollar el todo y no las partes por separado.

En consideración, el líder sistémico debe proyectar integrar el talento humano de la organización al logro de metas y objetivos comunes, para esto es indispensable que cada persona aporte la mayor de sus competencias, siendo esta una tarea primordial por parte del líder sistémico. A su vez, el líder sistémico debe desarrollar y emplear diversas competencias que le permitan ser modelo de trabajo sinérgico, ante lo cual, Senge (2006: 7), lo denomina disciplina "para una innovación en conducta humana, es preciso ver los componentes como disciplinas". Prosiguiendo con lo planteado, Senge (op. cit.) señala que existen cinco disciplinas que deben ser trabajadas de modo simultáneo para ser un líder sistémico, entre las cuales se hace mención:

1. Pensamiento sistémico: El pensamiento sistémico es un marco conceptual, un cuerpo de conocimientos y herramientas que se ha desarrollado en los últimos cincuenta años, para que los patrones totales resulten más claros, y para ayudarnos a modificarlos.

2. Dominio personal: El dominio personal es la disciplina que permite aclarar y ahondar continuamente nuestra visión personal, concentrar las energías, desarrollar paciencia y ver la realidad objetivamente. En cuanto tal, es una piedra angular de la organización inteligente, su cimiento espiritual. El afán y la capacidad de aprender de una organización no pueden ser mayores que las de sus miembros.

3. Modelos mentales: Los "modelos mentales" son supuestos hondamente 


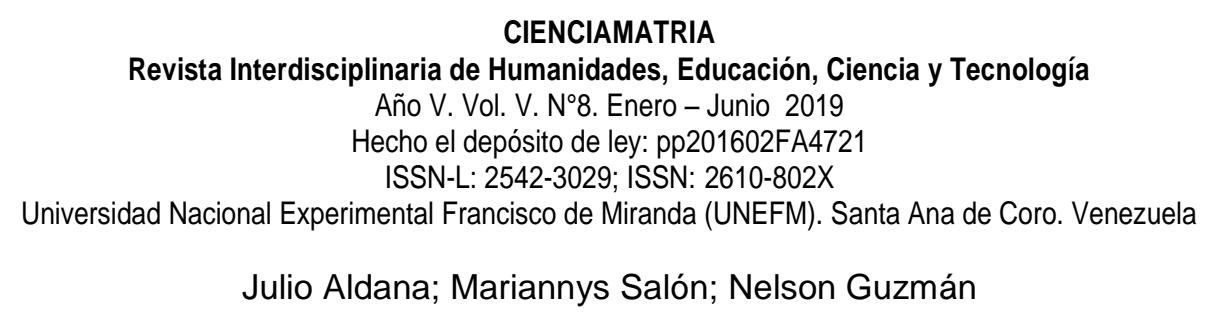

arraigados, generalizaciones e imágenes que influyen sobre nuestro modo de comprender el mundo y actuar. A menudo no tenemos conciencia de nuestros modelos mentales o los efectos que surten sobre nuestra conducta.

4. Construcción de una visión compartida: Si una idea sobre el liderazgo ha inspirado a las organizaciones durante miles de años, es la capacidad para compartir una imagen del futuro que se procura crear. Cuesta concebir una organización que haya alcanzado cierta grandeza sin metas, valores y misiones que sean profundamente compartidos dentro de la organización.

5. Aprendizaje en equipo: La disciplina del aprendizaje en equipo comienza con el "diálogo", la capacidad de los miembros del equipo para "suspender los supuestos" e ingresar en un auténtico "pensamiento conjunto".

Por consiguiente, el líder sistémico tiene el reto de integrar las cinco disciplinas descritas, para ser trabajadas sinérgicamente en la organización, de allí que no puede existir tal liderazgo sin el uso efectivo del pensamiento sistémico, por cuanto Senge (2006: 6) señala que

Por eso el pensamiento sistémico es la quinta disciplina. Es la disciplina que integra las demás disciplinas, fusionándolas en un cuerpo coherente de teoría y práctica. Sin una orientación sistémica, no hay motivación para examinar cómo se interrelacionan las disciplinas. Al enfatizar cada una de las demás disciplinas, el pensamiento sistémico nos recuerda continuamente que el todo puede superar la suma de las partes.

Desde la gerencia educativa, tanto en ambientes de aprendizajes como a nivel directivo, es un reto trabajar sistémicamente, por cuanto la cultura inherente a las instituciones, es referente al pensamiento conductual, el cual es contrario a lo planteado en el mundo sistémico. Sin embargo, la postura del pensamiento binario o mecánico, es inculcada consciente e inconscientemente en nuestras organizaciones educativas, encontrando refuerzos en la sociedad donde el líder de tipo vertical - jerárquico, aún tiene predominio, influenciando en los comportamientos sociales de las personas. Complementando la visión expuesta, Medina, Nava y Bong (2010: 8) señalan que 


\author{
CIENCIAMATRIA \\ Revista Interdisciplinaria de Humanidades, Educación, Ciencia y Tecnología \\ Año V. Vol. V. N8. Enero - Junio 2019 \\ Hecho el depósito de ley: pp201602FA4721 \\ ISSN-L: 2542-3029; ISSN: 2610-802X \\ Universidad Nacional Experimental Francisco de Miranda (UNEFM). Santa Ana de Coro. Venezuela \\ Julio Aldana; Mariannys Salón; Nelson Guzmán
}

El liderazgo del pensamiento sistémico, el liderazgo emergente, el liderazgo que busca el aglutinamiento de realidades, de lógicas, en factores comunes de entendimiento, en visiones comunes, que se soportan en valores, principios, virtudes y comunicación, con la finalidad de crear el clima armónico, un ambiente organizacional, que dé lugar a todas las transformaciones de pensamiento y actitudes necesarias, para el logro de los objetivos y llegar al futuro esperado por una sociedad.

Por lo tanto, la sociedad postmoderna retoma el diálogo como medio para crecer holísticamente a partir de las fortalezcas que cada persona pueda aportar a la organización, esto implica que cada talento humano, tiene la potencialidad para contribuir al desarrollo integral de la organización, tornándose un liderazgo compartido en donde cada uno cumple funciones diferentes pero con un mismo objetivo que alcanzar en la prosecución de una sociedad donde el ser humano sea el centro de la misma.

\title{
Competencias gerenciales en el docente universitario
}

El docente universitario tiene funciones de docencia, investigación y extensión, por consiguiente las competencias gerenciales que debe desarrollar para gerenciar con una visión sistémica, deben estar enfocadas hacia las vertientes mencionadas. Sin embargo, es necesario partir del término "competencias", en este sentido la Organización Panamericana de la Salud (2002: 1), define por competencia

La aptitud de un individuo para desempeñar una misma función productiva en diferentes contextos y con base en los requerimientos de calidad emprendidos por el sector productivo. Esta aptitud se logra con la adquisición y desarrollo de conocimientos, habilidades y capacidades que son expresadas en el saber, el hacer y el saber hacer, es decir, la capacidad real para lograr un objetivo o un resultado en un contexto dado.

Partiendo de lo planteado, el docente universitario debe desarrollar competencias en docencia, investigación y extensión, además de las gerenciales que le permitan dirigir la organización con un basamento en competencias, esto permitirá estructurar el trabajo 
organizado de la institución con el fin de generar sinergia entre los miembros de la organización en aras de trabajar hacia metas comunes. En tal sentido, las competencias pueden ser vistas como la integración de conocimiento, habilidades y actividades con la idea de gestionar las capacidades de los trabajadores hacia el logro efectivo de los planes propuestos. Complementando lo expuesto, Morles (2009:104) plantea que

El término competencia gerenciales, se entiende como la habilidad que posee un docente para sistematizar acciones y racionalizar recursos en los planteles. Visto desde el punto de vista gerencial, el concepto de competencia se refiere a la capacidad del gerente para alcanzar las metas propuestas para la escuela,

Por consiguiente, surge la necesidad de formar a los docentes en competencias gerenciales que permitan actuar de un modo efectivo en la interacción de las relaciones organizacionales en pro de lograr las metas propuestas pero con la efectividad necesaria, para esto se hace necesario que los docentes que hacen vida en la institución se integren a las actividades propuestas desde la gerencia con el fin de trabajar en equipo para el logro de objetivos comunes, para esto es necesario que el gerente educativo tenga cualidades que motiven a los docentes a integrarse y trabajar sinérgicamente, de lo contrario puede darse acciones negativas que imposibiliten el trabajo al logro común, produciéndose pérdidas para la organización.

En función de lo expuesto, es necesario conocer que existen diversos tipos de competencias, para Álvarez y Cárdenas (2001: 23), hay tres tipos fundamentales de competencias que conviene distinguir: Las competencias gerenciales y las competencias técnica o de puesto y las específicas; todas son imprescindibles en el ejercicio de cualquier responsabilidad gerencial por parte del docente universitario. En este sentido:

Las competencias técnicas, según Álvarez y Cárdenas suelen incluir conocimientos o habilidades específicas necesarias para desempeñar una tarea concreta. De allí que determinados puestos pueden requerir cierto dominio con otro idioma, manejo de 


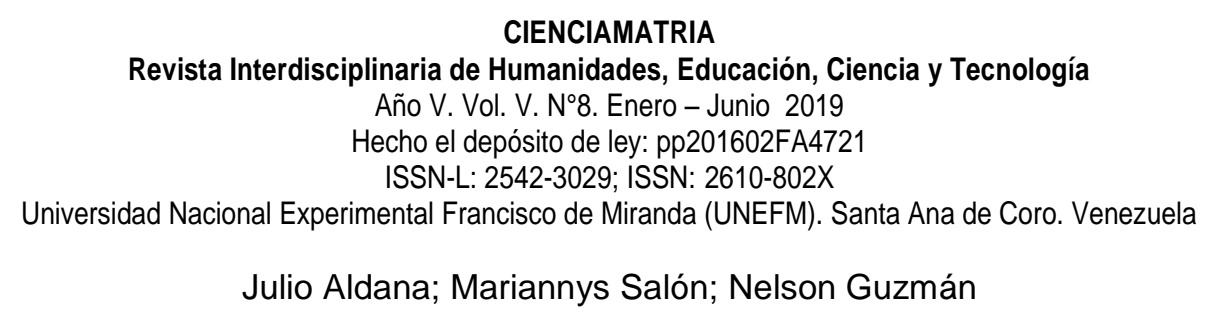

tecnología, manejo del estadístico y de investigación, lo cual eleva la capacidad resolutiva del director.

Las competencias gerenciales son aquellos comportamientos observables y habituales que posibilitan el éxito de una persona en función directiva. Estas competencias son más gerenciales y aunque cada institución pueda enfatizar más unas que otras, pueden estudiarse de manera conjunta a partir del análisis de la función educativa. Las competencias específicas son aquellas habilidades propias y vinculadas a una titulación, le dan identidad y consistencia profesional al perfil formativo del individuo.

Desde lo abordado, se genera la posibilidad de que cada institución universitaria pueda promover competencias gerenciales en el los docentes universitarios, esto tendría que ver con la filosofía de gestión y políticas de la misma, las cuales pueden direccionar hacia donde trabajar desde el enfoque gerencial, ciertamente como se plantea desde la visión por competencias, existen similitudes con otras universidades, sin embargo, lo especifico marca la pauta y la distancia, hacia donde se debe orientar el trabajo gerencial.

\section{REFERENCIALES METODOLÓGICOS}

La investigación se enmarca en el paradigma interpretativo, por cuanto se estudian los significados de las acciones humanas y de la vida social en relación al fenómeno de estudio, usando como criterio de evidencia, el acuerdo intersubjetivo en el contexto educativo. En este sentido, desde el paradigma interpretativo se sustituyen los ideales teóricos de explicación, predicción y control, por los de comprensión, significado y acción.

En consideración; para Sandín (2003: 56), el paradigma interpretativo "emergió como reacción al intento de desarrollar una ciencia natural de los fenómenos sociales", es decir, es un paradigma que comprendió desde las realidades sociales todos aquellos factores culturales, sociales, humanos, religiosos, entre otros, producto de una comunidad, a fin interpretar y comprender las realidades sociales. El paradigma 
interpretativo para González (2011: 16),

....aspira simplemente a explicar los significados subjetivos asignados por los actores sociales a sus acciones, así como a descubrir el conjunto de reglas sociales, que dan sentido a las actividades sociales sometidas a escrutinio sociales (en nuestro caso las actividades educativas), y así revelar la estructura de inteligibilidad que explica el porqué de dichas acciones, que tienen los sujetos que las emprenden.

Desde lo planteado, se comprende que el paradigma interpretativo parte de un entorno para interpretar las realidades vividas por los actores sociales desde el ambiente natural en que ocurren los eventos, es decir, se sumerge en la dinámica de vida de las personas, comprenden las acciones propias, su percepción y visión de la realidad, para interpretarla y darle significado.

El método de investigación, se orienta hacia el hermenéutico - fenomenológico por cuanto este permite estudiar, comprender, las vivencialidades desde la psiquis - acción de los actores sociales involucrados en el proceso investigativo, de ese modo, la interpretación de tales significados se teorizará en función del fenómeno de estudio. Para la construcción de este proceso dialectico - hermenéutico, se tienen en cuenta los postulados de Dilthey (1900) quien afirma "toda expresión de la vida humana es objeto natural de la interpretación hermenéutica".

Esto permitirá interpretar los significados escritos de las expresiones humanas que han comunicado una verdad en un determinado momento, haciendo posible que los investigadores internalicen las premisas emergentes con el fin de codificar información de entrada que pueda ser cotejada con la resultante del proceso investigativo. Por otro lado, Gadamer (1975: 67) afirma que

Nunca podremos tener un conocimiento objetivo del significado de un texto - de cualquier otra expresión de la vida psíquica, ya que siempre estaremos influidos por nuestra condición de seres históricos: con nuestros valores, normas y estilos de pensamiento y de vida, nunca tendremos conocimiento objetivo del significado de un texto o de cualquier otra cosa. La interpretación implica una "fusión de horizontes", una interacción dialéctica entre las expectativas de quien interpreta y el significado del texto o acto humano 


\section{CIENCIAMATRIA \\ Revista Interdisciplinaria de Humanidades, Educación, Ciencia y Tecnología \\ Año V. Vol. V. N8. Enero - Junio 2019 \\ Hecho el depósito de ley: pp201602FA4721 \\ ISSN-L: 2542-3029; ISSN: 2610-802X \\ Universidad Nacional Experimental Francisco de Miranda (UNEFM). Santa Ana de Coro. Venezuela \\ Julio Aldana; Mariannys Salón; Nelson Guzmán}

Esto permite que los investigadores, escruten lo planteado por los autores que fueron revisados como teoría de entrada, con el fin de cotejar la visión heurística con la onto epistémica, de ese modo, podrá existir un debate entre las ideas planteadas entre ambas visiones para dialogar en la utilidad de tener en cuenta la posición histórica sobre el fenómeno de estudio y el devenir que podría emerger sobre el mismo, por consiguiente para el procedimiento investigativo se tiene en consideración la perspectiva metodológica de Gadamer sobre las fases para acceder a lo estudiado, el cual se especifica a continuación:

Fase I. La interpretación: En esta fase se exponen las ideas y apreciaciones del investigador con respecto a los textos analizados, las entrevistas y las visiones de la investigadora realizadas tomando en consideración el contexto y tiempo en el que fueron realizados, así mismo, la interpretación funde lo aspirado por el intérprete y lo ofrecido significativamente por el texto o el acto humano.

Fase II. La comprensión: Esta fase incluye las entrevistas y conversaciones que lleven a la interpretación del fenómeno de estudio, todo ello para conocer cómo fue la emocionalidad que sintieron los informantes claves en su vivencialidad con el fenómeno de estudio. En este sentido, Gadamer (1975) afirma que está fase comprende el análisis comprensivo de las fuentes y su interconexión con las localidades problemáticas permitirá hacer congruente el hilo discursivo, en el razonamiento interpretativo y de aplicación de los contenidos a obtenerse.

Fase III. La aplicación: En esta se lleva a cabo el desarrollo de las ideas iniciales con base en lo extraído de los textos, entrevistas y reflexiones en este caso, se trata de la verificación de los objetivos trazados a través de la interpretación. Para Gadamer (1975) la aplicación no quiere decir aplicación ulterior de una generalidad dada, comprendida primero en sí misma a un caso concreto; ella es más bien la primera verdadera comprensión de la generalidad que cada texto dado viene a ser para nosotros: La comprensión es una forma de efecto, y se sabe a sí misma como efectuar. El diseño de investigación con el cual se trabaja es el cualitativo, ante lo cual, Sandín 
(2003: 138), considera que

El diseño de una investigación cualitativa exige que el investigador se posicione y adopte una serie de decisiones no solo durante la elaboración del proyecto de investigación y al inicio del proceso investigador, sino también durante y al finalizar el estudio. Es por ello que la mayoría de autores coinciden en identificar el proceso de investigación cualitativa como emergente, flexible, y no lineal, enfatizando su carácter contextual y de adaptación a la realidad y avatares de la propia investigación.

Las ideas expuesta por Sandín, permiten afirmar que el diseño de investigación cualitativa, el investigador se apropia del método y todo su proceso, a fin de tomar decisiones aceptadas para comprender la realidad de estudio durante el pre-inicio, inicio, desarrollo y culminación de la investigación. Por lo tanto, es considerado como un diseño emergente que surge de la interacción entre el investigador y los actores sociales, de esa interactividad entre el contexto social, y las diferentes situaciones presentes la investigación.

El trabajo científico asumirá el diseño de investigación cualitativa de campo por su carácter emergente, flexible y de adaptabilidad al contexto social, para comprender las realidades vividas por los informantes claves con el fenómeno de estudio con el fin de conocer sus vivencias en pro de consolidar el propósito principal de la investigación.

El diseño cualitativo, por ser emergente flexible, permite hacer un recorrido metodológico en base a la consecución de los propósitos secundarios de la investigación, ante lo cual, se tiene que se apoya en un nivel de investigación integrativo, explicado por Hurtado de Barrera (2005: 73), como "el conocimiento trasciende el campo de las explicaciones para expresarse en acciones concretas que, de manera intencional y planificada, modifican o transforman el evento que se está estudiando", es decir, es un nivel que trasformar una realidad de estudio, ofreciendo alternativa de solución sobre la problemática estudiada.

En atención al estudio, el nivel integrativo permite integrar visiones fenomenológicas hermenéuticas para la comprensión y teorización del fenómeno de estudio, logrando 
que el conocimiento científico trascienda más allá de un nivel cognitivo. Por lo tanto, el estudio se realizará teniendo como método principal la hermenéutica de Gadamer, bajo el nivel integrativo de la fenomenología de Heidegger, la cual implica íntimamente la hermenéutica. El nivel integrativo permite integrar aspectos hermenéuticos, fenomenológicos, como elementos propiciadores de un devenir metodológico que subyacen para lograr el propósito principal establecido por los investigadores, al gestar su relación ontológica con el fenómeno de estudio.

En relación a los informantes claves; Goetz y Lecompte (1988: 134), señalan que son "individuos en posesión de conocimiento, status o destrezas comunicativas especiales y que están dispuestos a cooperar con el investigador". Son personas que hacen vida en el escenario de investigación, tienen una relación ontológica con el fenómeno de estudio en similitud a la investigadora y sobre todo; poseen información relevante para el proceso investigativo. Por otro lado; Goetz y Lecompte (1988: 134), señalan que los informantes claves son "personas residentes durante mucho tiempo en una comunidad, miembros de instituciones fundamentales o conocedores de los ideales culturales del grupo", es decir, personas con alto grado de conocimiento sobre el fenómeno que se investiga.

De ese modo, se tiene que los informantes claves, están constituidos por cinco actores sociales que hacen vida activa en la Universidad Nacional Experimental Francisco de Miranda (UNEFM). A su vez, los informantes claves serán codificados a fin de contar con un proceso metódico a la hora de analizar la información aportada, tal codificación está conformada por un número y dos letras del nombre del informante clave.

\section{ALCANCES ESPERADOS POR LA INVESTIGACIÓN}

La presente investigación se encuentra en construcción, siendo su primer alcance el "generar reflexiones sobre los hallazgos del liderazgo sistémico del docente como eje integrador de las competencias gerenciales en la Universidad Nacional Experimental Francisco de Miranda", esto con la intención de iniciar progresivamente la teorización 
(segundo alcance) sobre lo planteado, quedando en este primer compendio investigativo lo relacionado al propósito hermenéutico - fenomenológico, lo cual permitirá tener un producto científico que sirva de base gnoseológica en la estructuración de un conocimiento que pueda ser sometido al mundo académico científico del campo educativo universitario, con la finalidad de generar a posteriori una teoría que permita contribuir en gestionar praxis gerenciales concomitantes con los nuevos enfoques de pensamiento de la sociedad postmoderna que invitan al aprender haciendo a lo largo de la vida.

\section{REFLEXIÓN INICIAL}

La sociedad globalizada de las dos últimas décadas ha conducido al mundo empresarial a reinventarse para poder ser competitivo, eficaz, productivo, ofrecer calidad a sus clientes. No obstante, en el contexto educativo por los nuevos paradigmas como la complejidad de Morin, entre otros, se encuentra en una metamorfosis donde debe empoderarse de nuevos estilos de aprendizaje, planificación, evaluación, investigación, gerencia, liderazgo, para estar en concordancia con los retos epistémicos de las instituciones postmodernas del conocimiento.

La praxis gerencial educativa busca establecer nuevos modos de liderazgo docente, para generar un proceso educativo en consonancia con las actuales tendencias gerenciales en donde se habla de organizaciones inteligentes que aprenden haciendo, en donde se hace mayor énfasis en las potenciales de las personas para que cada una pueda aportar lo mejor de sí al éxito de la organización, situación que implica que las instituciones se actualizan constantemente y se mantengan en la vanguardia gerencial, esto conlleva a una práctica donde la introspección del ser educativo en reorientarse como persona - profesional, se encuentra en la cúspide para comprender el devenir educativo de los nuevos tiempos, configurándose en el hacer más que en el saber.

En este sentido, la Organización de las Naciones Unidas para la Educación, la Ciencia y la Cultura (UNESCO: 2015), ha visionado la educación 2030, la cual tiene como 
objetivo "asegurar una educación inclusiva, equitativa y de calidad, así como promover oportunidades de aprendizaje para todas las personas a lo largo de la vida". Tal promoción se basa en transformar el liderazgo educativo tradicional, donde se procede a enseñar en el aula de clases mediante una relación epistémica sujeto - objeto.

Desde lo planteado por la UNESCO, la praxis gerencial educativa debe enfocarse en el sujeto - sujeto donde ambos construyen conocimiento, más aun, debe involucrarse una perspectiva sistémica de aprendizaje para analizar las partes que conforman el todo. De ese modo, la educación abandona los espacios tradiciones de enseñanza para desarrollarse en la gran aula global que es el mundo.

Según el párrafo anterior, la interconexión de eventos debe configurar la apertura de la mente para entender que nada en el mundo postmoderno sucede de modo aislado, sino que existen causas - efectos, donde la toma de decisiones en ámbitos diversos a la educación pueden influir en ella, como por ejemplo: la política, la economía, entre otros. Comprender esta conexión promueve un nuevo estilo de pensar y actuar en las organizaciones educativas y específicamente en las universidades, es decir, un nuevo líder. Ante lo cual, Deming (2012: 12) señala que

El pensador sistémico busca comprender a cambio de culpar, ya que sabe que las culpas traen consecuencias negativas para la organización y la gente. La comprensión de la dinámica de la organización en comparación con su visión de futuro es el objetivo del pensamiento sistémico. El aprendizaje es el objetivo principal, ya que sin aprendizaje estamos condenados a hacer lo mismo y a obtener lo mismo. El pensamiento lineal es lo opuesto al pensamiento sistémico. Pensamos linealmente cuando buscamos culpables o cuando, en general, buscamos la causa inmediata de algún evento.

El líder desde la perspectiva del pensamiento sistémico, debe visualizar las capacidades de la persona para motivarla al máximo potenciamiento de la misma. En este sentido, entra en juego la divergencia del aprendizaje, ante lo cual, no puede enseñarse de modo lineal, por cuanto unos individuos tendrán potencialidades para aprender - hacer unas cosas y otras no. De allí que no puede hablarse de negatividades, sino de potencialidad en la persona, siendo esta la tarea primordial del 


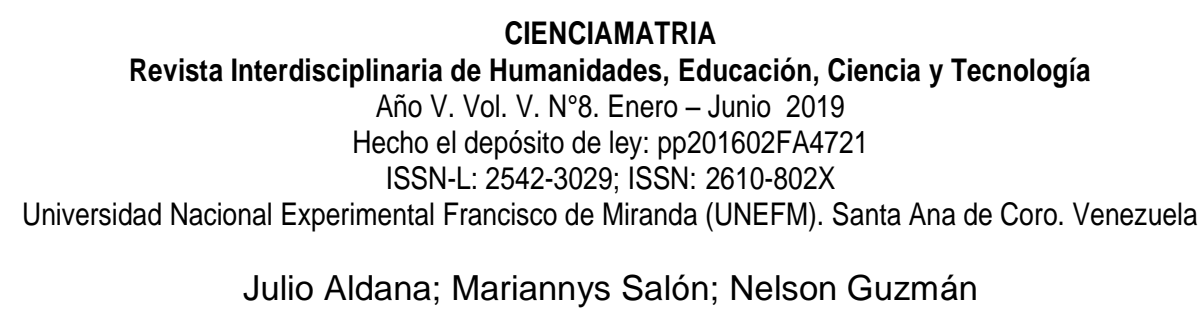

líder sistémico, desde tal visión el docente busca descubrir en sí mismo y en los estudiantes, las oportunidades para crecer integralmente desde una mirada sistémica del mundo. En este sentido, Medina y Nava (2010:1) señalan que liderazgo sistémico

Concibe al hombre como núcleo fundamental del sistema, y la organización como un sistema social, con interacción del entorno, para el desarrollo sustentable. Un liderazgo que diseña, educa y sirve, soportados sobre la visión compartida, valores y comunicación ética, como base del cambio del pensamiento de las organizaciones del siglo XXI, que caracterizan el liderazgo sistémico, como ente sincronizador de acciones y actividades.

El docente como líder sistémico, tiene la posibilidad de crear y recrear la praxis educativa en un acto donde las habilidades de los estudiantes son potenciadas al máximo para construir un aprendizaje que no solo sea significativo, sino transformador de la persona y del ecosistema donde éste habita. Complementando la idea planteada, Medina y Nava (2010:5) exponen que

Para el desarrollo integral de las organizaciones, de un pensamiento holístico e integrador y de esta manera romper el cordón que une al pensamiento fragmentador y mecanicista todavía presente. Esta es la lógica que debe gobernar al liderazgo emergente, este es el enfoque del Pensamiento Sistémico, el cual va más allá del hombre como función de comportamiento lineal y determinista.

Desde esta visión, el líder sistémico debe promover la comunicación asertiva como elemento no solo comunicativo, sino motivador de la persona, estableciendo una relación intersubjetiva que procura la construcción de conocimiento mediante como punto de interconexión del estudiante con el mundo, estableciéndose un diálogo donde el significado otorgado a la palabra tiene poder para construir la razón, la cual debe ser llevada a la práctica para establecer una educación holística e integradora de los procesos mentales y pragmáticos del ser humano en procura de establecer principios de efectividad y calidad en pertinencia a la dinámica de vida en la cual se encuentra inmerso.

El docente (bien sea en rol de aula o gerencial) al desarrollarse como líder sistémico, debe enfocarse en superar en sí mismo y en los estudiantes, los esquemas mentales 


\section{CIENCIAMATRIA \\ Revista Interdisciplinaria de Humanidades, Educación, Ciencia y Tecnología \\ Año V. Vol. V. N8. Enero - Junio 2019 \\ Hecho el depósito de ley: pp201602FA4721 \\ ISSN-L: 2542-3029; ISSN: 2610-802X \\ Universidad Nacional Experimental Francisco de Miranda (UNEFM). Santa Ana de Coro. Venezuela \\ Julio Aldana; Mariannys Salón; Nelson Guzmán}

lineales que se interponen para proyectar, ejercer, una praxis educativa sistémica en concordancia con la transformación social de su entorno. Es en este punto donde el trabajo en equipo debe desarrollarse para construir en conjunto las nuevas realidades educativas - sociales del ecosistema de vida humana que han de converger en una educación liberadora, transformadora, de los actores involucrados en la misma. Por consiguiente Medina y Nava (2010:137) expresan que

El liderazgo sistémico se fundamenta, en la integración, en la comunicación, en el conocimiento y la aplicación de elementos compartidos por todos, es la cualidad de influenciar en los grupos sociales, a través de la visión común, motivación, aprendizaje, comunicación, para lograr los objetivos trazados por todos los que conforman la organización. Es un modelo ético y de comportamiento asertivo de liderazgo moderno y emergente.

La cita de Medina y Nava, invita a transcender hacia un agente de cambio, un auténtico líder, capaz de movilizar su energía, generando una actitud y espíritu productivo que actúe como promotor de un liderazgo compartido, Propiciando así, el trabajo en equipo, un ambiente armónico de colaboración, comunicación y motivación, que permita el alcance de las metas, el rendimiento del grupo y la satisfacción de las necesidades institucionales, escalando hacia la educación por competencias, a lo holístico, entre otros modelos o enfoques, para que cada persona trabaje, construya, aporte, según la visión e inteligencia que pueda tener en servicio del crecimiento integral de la sociedad. En concordancia con lo expuesto hasta ahora, se narra la relación ontológica entre los investigadores y el fenómeno de estudio, es decir el liderazgo sistémico. Por cuanto desde el ejercicio docente en la Universidad Nacional Experimental Francisco de Miranda (UNEFM), surge la preocupación manifiesta de un grupo de docentes por transcender al modelo actual de liderazgo y gerencia que se implementa en la UNEFM. Es de destacar que actualmente se trabaja (UNEFM) con el modelo instruccional de aprendizajes, es decir, con diseños instruccionales en donde el docente pre concibe no solo los contenidos a transmitir en el aula de clases, sino, la conducta que deben tener los estudiantes, de ese modo, se espera un comportamiento y aprendizaje homogéneo 


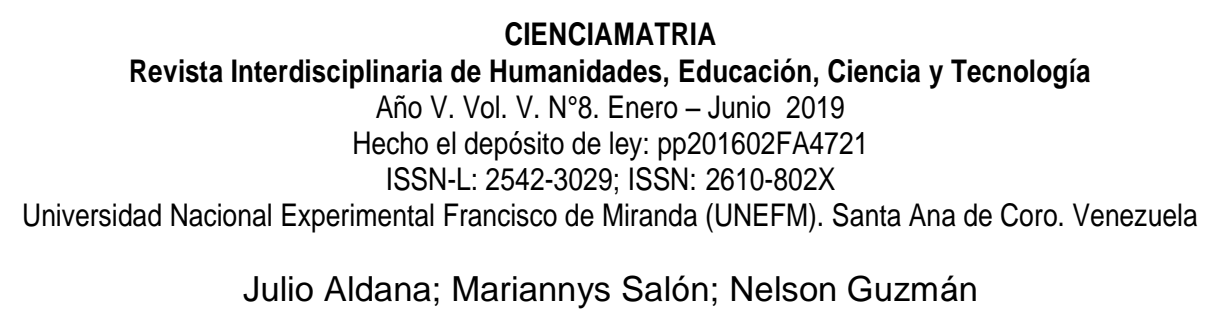

en todos los estudiantes, siendo esto distante de la realidad, por cuanto cada estudiante por ser una persona, tiene intereses, valores, cultura, modo de aprender, entre otras cualidades y aptitudes, diferentes al de sus compañeros.

Esto origina la "educación bancaria" o mecanicista, es decir, en donde el docente dicta en mayor proporción clases magistrales y los estudiantes simplemente son calificados como buenos o malos en la medida que aprueban o no, los exámenes estándares para medir el conocimiento, así como otras evaluaciones propias del modelo instruccional.

Al trabajarse casi exclusivamente con una visión basada en el diseño instruccional, donde se les enseña a los estudiantes mediante estrategias que buscan fomentar el conocimiento, sin tener mayor contacto con la práctica, es decir, se desaprovecha el potencial de los estudiantes y del entorno social donde se encuentra la UNEFM, evitándose el uso con mayor frecuencia de actividades que permitan "aprender haciendo", aprendizaje por proyecto, por competencias, entre otros enfoques, en donde se favorece una educación holística, basada en mayor contacto directo del estudiante con su campo o área de trabajo como futuro profesional.

Ahora bien surge la interrogante sobre ¿la relación del liderazgo sistémico con el diseño instruccional? Simplemente al trabajar en mayor frecuencia con el diseño instruccional, el liderazgo sistémico no fluye en todo su potencial, es decir, lo instruccional obedece a una visión de liderazgo sujeto - objeto y no al sujeto - sujeto, siendo visiones epistémicas gerenciales distantes una de otra.

Por consiguiente, la cultura sobre el liderazgo docente y gerencial educativa en la UNEFM se desarrolla desde el sustento de enfoques de aprendizaje que en la actualidad no brindan las repuestas necesarias que la sociedad demanda para su transformación y progreso integral, se hace necesario gestar un nuevo modo de comprender el mundo educativo, proyectando desde allí, aprendizajes que permitan en el mediano plazo, contar con profesionales altamentes calificados no solo para conocer, sino, para hacer, no solo en su campo de trabajo, sino, en contribuir en cambios favorables para la sociedad venezolana. 


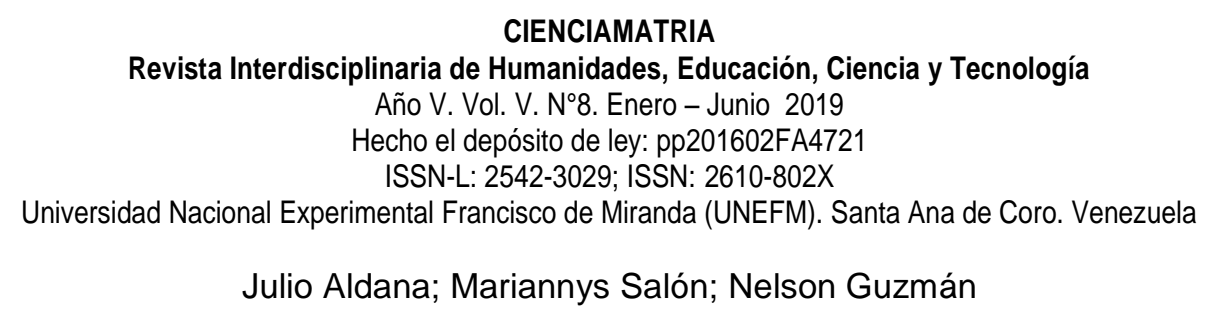

Prosiguiendo con lo planteado, se hace necesario transcender a nuevos modelos de liderazgo - gerenciales educativos que impulsen una praxis educativa que se encuentre en concordancia con los lineamientos teóricos, políticas educativas nacionales e internacionales, pero que sobre todo transcienda hacia lo sistémico para trabajar con una visión integral, donde las capacidades de los actores involucrados permitan reaprender y empoderarse de una nueva praxis docente gerencial, donde la introspección sobre el ser docente sea el punto reflexivo para iniciar un camino hacia la consecución del liderazgo sistémico como principio accionario de una educación transformadora.

Es importante transcender la visión actual gerencial educativa con la cual se trabaja en la UNEFM, hacia la de líder sistémico, es decir, hacia la gerencia educativa basada por competencias desde la vertiente sistémica, por cuanto esto permite trabajar en la formación de estudiantes no solo acumuladores de conocimiento, sino aptos para el hacer y aprender a lo largo de la vida, así como el docente se constituye en un líder polivalente, incentivando el progreso integral del estudiante y de la universidad en sintonía con las exigencias de la sociedad actual.

Para lograr lo planteado, el estilo de liderazgo gerencial educativo, debe dar un giro para motivar al docente a promover una educación sistémica - holística, que forme para los retos del mundo del tercer milenio, es decir, formar a estudiantes en capacidad de tomar decisiones asertivas, ser proactivo, emprendedor, innovador, líder transformacional - sistémico, en este sentido la Organización para la Cooperación y el Desarrollo Económico (OECD) (2016: 15) plantea que

Hay una creciente preocupación con respecto a que la función del director escolar, diseñada para la era industrial, no ha evolucionado para tratar los complejos desafíos para los cuales las escuelas preparan a niños y jóvenes a enfrentar en el siglo XXI

Por lo tanto, el liderazgo y la gerencia, con el cual se trabaja en la UNEFM afecta directa e indirectamente en la adaptación social - laboral que deben desarrollar los 


\section{CIENCIAMATRIA \\ Revista Interdisciplinaria de Humanidades, Educación, Ciencia y Tecnología \\ Año V. Vol. V. N8. Enero - Junio 2019 \\ Hecho el depósito de ley: pp201602FA4721 \\ ISSN-L: 2542-3029; ISSN: 2610-802X \\ Universidad Nacional Experimental Francisco de Miranda (UNEFM). Santa Ana de Coro. Venezuela \\ Julio Aldana; Mariannys Salón; Nelson Guzmán}

futuros egresados en la real y dinámica sociedad actual, debido que es producto de una praxis educativa gerencial que no se encuentra en sintonía con las exigencias hacia donde se enfoca la educación mundial, sustentada desde la visión de la UNESCO hacia la educación 2030, es por ello que los sistemas educativos han ido adoptando el enfoque por competencias con el fin de formar integralmente a las personas y puedan desenvolverse de un mejor modo en la sociedad. Es así que OECD (2016: 15) señala que

Al graduarse, los niños y los jóvenes en las escuelas de todo el mundo se enfrentarán a un futuro muy diferente del de las generaciones anteriores. Los avances tecnológicos y los descubrimientos científicos están acelerando de manera significativa la cantidad de conocimiento e información disponible. Ahora vivimos en una comunidad internacional cada vez más interdependiente, en la que el éxito o el fracaso de un país tiene consecuencias para muchos otros.

La UNEFM, debe garantizar que sus egresados, no solo obtengan buena información durante su proceso educativo, sino que tengan las competencias necesarias para ser competentes en el campo laboral, emprendedores, innovadores, para gestionar una praxis profesional en concordancia con el pensamiento y liderazgo sistémico, de ese modo, se garantiza la posibilidad de contar con ciudadanos activos, reflexivos, del rol protagónico que tienen para transformar la sociedad.

Es allí donde el ejercicio docente gerencial realizado en la UNEFM, contribuye a reflexionar sobre cuál es el liderazgo ejercido en favor de una real y profunda transformación educativa en el contexto universitario, evidenciándose la necesidad de transcender al liderazgo sistémico y competencias gerenciales educativas, como alternativas para promover un accionar pedagógico en concordancia con las exigencias, retos, del nuevo milenio. La praxis docente no puede estar enmarcada solo en un proceso binario del conocimiento, debe transcender hacia el desarrollo de competencias gerenciales que permitan gestionar un conocimiento holístico, proveedor de capacidades para el ejercicio profesional con pertinencia en la transformación social 


\section{CIENCIAMATRIA \\ Revista Interdisciplinaria de Humanidades, Educación, Ciencia y Tecnología \\ Año V. Vol. V. N8. Enero - Junio 2019 \\ Hecho el depósito de ley: pp201602FA4721 \\ ISSN-L: 2542-3029; ISSN: 2610-802X \\ Universidad Nacional Experimental Francisco de Miranda (UNEFM). Santa Ana de Coro. Venezuela \\ Julio Aldana; Mariannys Salón; Nelson Guzmán}

necesaria para el progreso de la nación.

¿Cómo desarrollar en los docentes competencias sobre el liderazgo sistémico como elemento gerencial emergente en las instituciones educativas venezolanas? Es una pregunta que posiblemente encuentre repuesta en el devenir de la presente investigación, en donde se proyecta comprender el accionar sistémico del líder docente para encausar a una progresiva teorización de argumentos que permitan contar con un corpus teórico coadyuvante en transcender a un modelo educativo que permita formalizar el aprendizaje en un ente promotor de un humanismo complejo al servicio del aprender a hacer como pedagogía innovadora en la UNEFM. Es así que Franco (2016: 1) señala que

Los líderes más efectivos son los que piensan y actúan de forma sistémica, observando el panorama general en vez de dividirlo en partes aisladas, y los que tienen en cuenta a las personas como centro y como única ruta hacia un mejor desempeño

Esto involucra una ruptura epistémica en el modo en cómo se realizan actualmente los procesos educativo - gerencial en la UNEFM, para encontrarse frente a la posibilidad de generar un encuentro educativo en donde el docente sea un líder sistémico, constituyéndose en un agente de cambio transformador desde una perspectiva que invita a ir más allá del pensamiento y accionar lineal con el cual se ha manejado la educación universitaria hasta ahora, es necesario transcender el modelo lineal, en este sentido, (Gutiérrez, Rodríguez e Isea, 2017: 232), plantea que "Por lo tanto, si se sigue con este modelo pedagógico no se podrá dar respuesta oportuna a las necesidades sociales, ni mucho menos a las exigencias del mercado laboral actual".

Es la oportunidad para entender que todo docente es un líder, un gerente, dentro o fuera de los ambientes de aprendizaje, se encuentre o no en cargos directivos en la universidad, para esto es necesario enfocarse desde el liderazgo sistémico, por cuanto este permite gestionar acciones integrales en pro de alcanzar metas comunes en una dimensión basada en el ganar - ganar de los actores involucrados en el proceso 


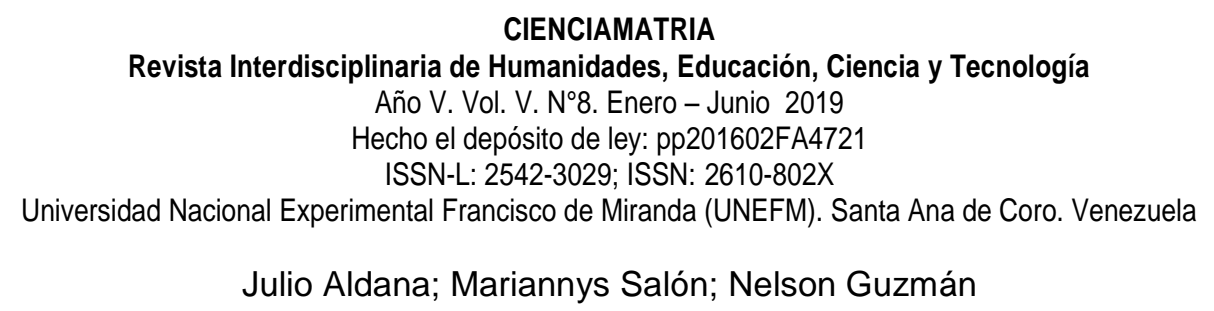

educativo gerencial de la universidad.

\section{REFERENCIAS CONSULTADAS}

1. Caldera, J. (2012). Cambio organizacional inteligente en los programas de postgrado de la Universidad del Zulia. Tesis doctoral. URBE. Maracaibo, Venezuela.

2. Deming, W. E. (2012). La nueva economía: para la industria, el gobierno y la educación. Díaz de Santos, Madrid, España.

3. Dilthey, W., "The rise of hermeneutics", (1900). En: Connerton, P. (ed), Critical sociology, Penguin, Nueva York.

4. Franco. C. (2016). Los líderes más efectivos son los sistémicos. [Artículo en línea]. Disponible: http://www.tendencias21.net/Los-lideres-mas-efectivos-sonlos-sistemicos_a4107.html.

5. Gadamer, H. (1975). La verdad y el método. Santiago de Chile. Universidad de Salamanca. España.

6. Gadamer (1988) Verdad y método: Fundamentos de una hermenéutica filosófica. Salamanca, España. Editorial Sígueme.

7. García, I. (2010). Liderazgo distribuido, una visión innovadora de la dirección escolar: una perspectiva teórica. Omnia Año 16, No. 3, 2010, pp. 19 - 36 ISSN: 1315 - 8856. [Artículo en línea]. Disponible: http://www.produccioncientifica.luz.edu.ve/index.php/omnia/article/view/7329/731 8

8. Galué, L. (2014). Liderazgo del supervisor en el desarrollo de la autonomía institucional en educación básica. Maestría en supervisión educativa en la Universidad Rafael Urdaneta, Maracaibo, Venezuela. Tesis / 9217-05 / 00068

9. Goetz, J. y LeCompte, M. (1988). Etnografía y diseño cualitativo en investigación cualitativa. Madrid-España. Edición Morata.

10.Gonzales, O. y Gonzales, L. (2012). Estilos de liderazgo del docente universitario. MULTICIENCIAS, Vol. 12, № 1, 2012 (35 - 44). ISSN 1317-2255 / Dep. Legal pp. $200002 \mathrm{FA} 828$ 


\begin{abstract}
CIENCIAMATRIA
Revista Interdisciplinaria de Humanidades, Educación, Ciencia y Tecnología

Año V. Vol. V. N8. Enero - Junio 2019

Hecho el depósito de ley: pp201602FA4721

ISSN-L: 2542-3029; ISSN: 2610-802X

Universidad Nacional Experimental Francisco de Miranda (UNEFM). Santa Ana de Coro. Venezuela

Julio Aldana; Mariannys Salón; Nelson Guzmán
\end{abstract}

11. González, J. (2011). El Paradigma Interpretativo en la Investigación social Educativa. Nuevas Respuestas Para Viejas Interrogantes. Tesis doctoral. Universidad de Sevilla. España.

12. Gutiérrez, R. (2014). Aproximación teórica a un modelo sistémico de gerencia educativa aplicable a los liceos adscritos al distrito escolar ํo 2 del Estado Cojedes. Tesis de maestría en gerencia avanzada en educación. Universidad de Carabobo, Venezuela.

13. Gutiérrez, N., Rodríguez, N., \& Isea, J. (2017). La emocionalidad frente a la innovación curricular. Revista Arbitrada Interdisciplinaria Koinonía, 2(3), 226-246. Recuperado de http://fundacionkoinonia.com.ve/ojs/index.php/revistakoinonia/article/view/61/48

14. Martínez, M. (2008). Epistemología y metodología cualitativa en las Ciencias Sociales. México. Editorial Trillas.

15. Medina, C., Nava, A., y Bong, I. (2010). Enfoque del liderazgo del pensamiento sistémico en las organizaciones. [Artículo en línea]. Disponible: http://www.redalyc.org/articulo.oa?id=90415607004 [Consulta: 2016. Julio 24]

16. Medina, C. Nava, A. y Bong, I. (2010). Enfoque del liderazgo del pensamiento sistémico en las organizaciones. MULTICIENCIAS, Vol. 10, № 2, 2010 (133 138). ISSN 1317-2255 / Dep. Legal pp. 200002FA828

17. Montero, R. (2006). Liderazgo del supervisor de educación inicial, desde la perspectiva de la inteligencia emocional. Maestría en supervisión educativa en la Universidad Rafael Urdaneta, Maracaibo, Venezuela. Tesis / 9217-06 / 00039

18. OECD (2016). Mejorar el liderazgo escolar. [Artículo en línea]. Disponible: https://www.oecd.org/edu/school/43913363.pdf. [Consulta: 2016. Julio 26]

19. Pirela de Piña (2012). Liderazgo gerencial y desempeño del supervisor en las escuelas de la parroquia Germán Ríos Linares. Maestría en gerencia educativa en la Universidad Rafael Urdaneta, Maracaibo, Venezuela. Tesis / 9217-06 / 00039

20. Sandín, P. (2003). Investigación Cualitativa en Educación. Fundamentos y Tradiciones. Madrid-España. Editorial McGraw-Hill Interamericana de España, S.A.U.

21. UNESCO (2015). Foro mundial sobre la educación. [Documento en línea]. 


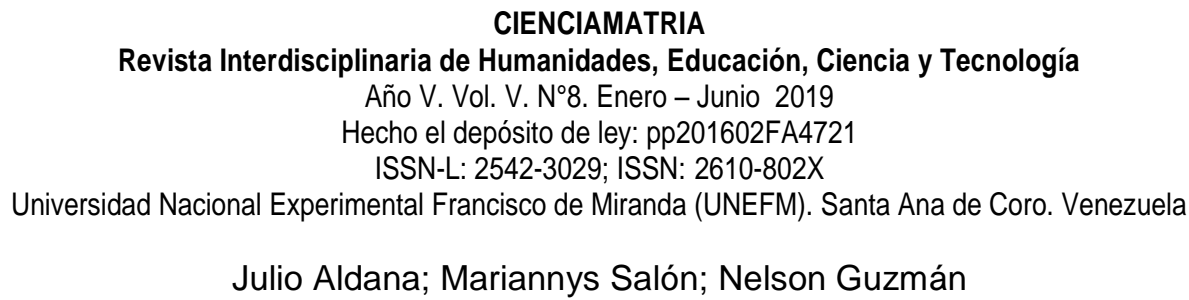

Disponible: $\quad$ http://es.unesco.org/world-education-forum-2015/aboutforum/declaracion-de-incheon

22. Valbuena, M. (2001). Teoría de Peter Senge y la organización educativa como sistema descentralizado. [Artículo en línea]. Disponible: http://www.produccioncientifica.luz.edu.ve/index.php/omnia/issue/view/852.

(C2019 por el autor. Este artículo es de acceso abierto y distribuido según los términos y condiciones de la licencia Creative Commons Atribución-NoComercial-Compartirlgual 4.0 Internacional (CC BY-NC-SA 4.0) (https://creativecommons.org/licenses/by-nc-sa/4.0/). 